\title{
Genetische Ursachen und Therapie der Osteogenesis imperfecta
}

\section{Pathophysiology and therapeutic options in osteogenesis imperfecta}

\section{(ㄷ)(1) (우) $\odot$}

Autoren

Oliver Semler ${ }^{1}$, Uwe Kornak ${ }^{2}$, Ralf Oheim ${ }^{3}$, Lothar Seefried ${ }^{4}$

Institute

1 Universität zu Köln, Medizinische Fakultät und Uniklinik Köln, Klinik und Poliklinik für Kinder- und Jugendmedizin, Köln, Deutschland

2 Institut für Humangenetik, Universitätsmedizin Göttingen, Germany

3 Universitätsklinikum Hamburg-Eppendorf, Institut für Osteologie und Biomechanik, Hamburg, Deutschland

4 Experimentelle und Klinische Osteologie, Lehrstuhl Orthopädie und Orthopädische Klinik König-Ludwig-Haus, Würzburg, Deutschland

Schlüsselwörter

Osteogenesis imperfecta, Therapie, Pathophysiologie, Bisphosphonate

Keywords

Osteogenesis imperfecta, Therapy, Pathophysiology, Bisphosphonates

\section{Bibliografie}

Osteologie 2020; 29: 302-310

DOI 10.1055/a-1233-9812

ISSN 0720-4299

(C) 2020. The Author(s).

This is an open access article published by Thieme under the terms of the Creative Commons Attribution-NonDerivative-NonCommercial-License, permitting copying and reproduction so long as the original work is given appropriate credit. Contents may not be used for commercial purposes, or adapted, remixed, transformed or built upon.

(https://creativecommons.org/licenses/by-nc-nd/4.0/)

Korrespondenzadresse

PD Dr. Oliver Semler

Klinik für Kinder- und Jugendmedizin,

Uniklinik Köln

Kerpener Straße 62

50931 Köln

Deutschland

Tel.: +49-221-478 84747

Fax: +49-221-478 1422361

E-Mail: joerg.semler@uk-koeln.de

\section{ZUSAMMENFASSUNG}

Die Osteogenesis imperfecta (OI) ist die häufigste angeborene Erkrankung, die zu einer erhöhten Fragilität des Skelettsystems führt. Die Variabilität des Phänotyps kann nicht vollständig durch die ursächlichen genetischen Veränderungen erklärt werden. Die meisten Formen werden durch Mutationen in Genen verursacht, die die Kollagen-Synthese/Prozessierung verändern, wobei die meisten Patientinnen und Patienten von Mutationen in den Genen COL1A1/A2 betroffen sind. Das bessere Verständnis der Pathophysiologie bei seltenen rezessiven Formen der Erkrankung hat bereits in zwei Fällen zu neuen therapeutischen Ansätzen geführt. Bei OI Typ VI, verursacht durch Mutationen in SERPINF1, kommt es zu einer Überaktivierung von Osteoklasten über den OPG/RANKL-pathway. Hier konnte gezeigt werden, dass eine Behandlung mit dem Osteoklasten-Antikörper Denosumab effektiver ist als eine antiresorptive Therapie mit Bisphosphonaten. Bei Patientinnen und Patienten, bei denen die ursächliche Mutation im Gen WNT1 liegt, bietet sich eine osteoanabole Behandlung mit Antisklerostin-Antikörpern an. Neben der medikamentösen Therapie sind die Bereiche der Rehabilitation und Orthopädie unverzichtbare Bestandteile einer interdisziplinären Behandlung.

\footnotetext{
ABSTRACT

Osteogenesis imperfecta (OI) is the most common hereditary disease causing an increased bone fragility. The wide variability of the phenotype can not be explained by the genotype. Most forms are caused by mutations in genes influencing synthesis and posttranslational modification of collagen. Most patients are affected by mutations in the genes COL1A1/A2. The increased knowledge about underlying genetic alteration and pathophysiology in some rare recessive forms has led to new therapeutic strategies. OI VI is caused by mutations in SERPINF1 leading to an increased activity of osteoclasts via the OPG/RANKL-pathway. In these patients a medical treatment with denosumab has been proven to be more effective as antiresorptive treatment compared to the former therapy with bisphosphonates. In patients affected by mutations in WNT1 an osteoanabolic treatment
} 
might be more suitable than an antiresorptive therapy. Pharmacological treatment has to be included in an interdisciplinary therapeutic concept in combination with physiotherapy and surgical treatment.

\section{Einleitung}

Die Osteogenesis imperfecta (OI) gehört zu den seltenen Erkrankungen mit einer Häufigkeit von ca. 1 : 20000. Das früher aufgrund der Klinik klar definierte Krankheitsbild hat sich in den vergangenen Jahren zu einer Gruppe von Erkrankungen gewandelt, die durch einen ähnlichen Phänotyp bei sehr heterogenen genetischen Ursachen gekennzeichnet ist. Die Ol ist die häufigste angeborene, genetisch bedingte Erkrankung, die zu einer reduzierten Stabilität des Skelettsystems führt. Das dominierende Symptom bei allen Erkrankungen aus diesem Formenkreis ist die erhöhte Anfälligkeit für Frakturen bei geringen Traumata. Die Frakturrate ist besonders hoch während der Kindheit und Jugend, also während des Körperlängenwachstums. Nach abgeschlossenem Längenwachstum kommt es bei den meisten Patientinnen und Patienten zu einer Phase mit relativ wenigen skelettalen Problemen. Im höheren Lebensalter können dann wieder vermehrt Frakturen, Knochenschmerzen und weitere Probleme des Skelettsystems auftreten [1].

Die meisten Formen der OI beruhen auf genetischen Veränderungen, die die Kollagen-Synthese und Kollagen-Modifikation betreffen. Abhängig von der Lokalisation der Störung in der Kollagenentstehung und -prozessierung treten neben den skelettalen Symptomen auch weitere Veränderungen in Geweben auf, die reich an Kollagen sind. Hierzu zählen z.B. der Bandapparat (Hypermobilität), eine unzureichende Adaptation der Gehörknöchelchen (Schallleitung-Schwerhörigkeit), eine Blauverfärbung der Skleren durch eine dünne Sklera mit verändertem Index der Lichtbrechung sowie eine Beteiligung der Zähne durch eine veränderte Dentin-Struktur [2].

Diagnostisch führend sind die skelettalen Symptome, die neben der Brüchigkeit der langen Röhrenknochen auch aus Deformierungen und Verkürzungen der Knochen bestehen können (Antekurvation der Unterschenkel, Verkürzung der Oberschenkel). Ein dysproportionierter Kleinwuchs unterschiedlicher Schwere kann bei den Patientinnen und Patienten durch Veränderungen der Wirbelkörper im Sinne von Sinterungen/Frakturen oder Fischwirbelbildung bedingt sein. Basierend auf den Veränderungen der Wirbelkörper, zusammen mit der Schwäche der Muskulatur, entwickelt sich bei vielen Betroffenen in der Pubertätsphase eine Skoliose [3].

Die Ausprägung der Erkrankung ist sehr variabel und kann von Verlaufsformen, die bereits pränatal aufgrund von intrauterinen Frakturen oder Verkürzungen der Röhrenknochen diagnostiziert werden, bis zu milden Verlaufsformen reichen, bei denen es lediglich in Phasen eines raschen Körperlängenwachstums zu Knochenbrüchen kommt.

Die Behandlung der OI ist überwiegend symptomatisch und sollte bei dem komplexen Symptombild der Erkrankung einen multidisziplinären Ansatz verfolgen. Hierbei ist neben der medikamentösen und orthopädischen Behandlung des Skelettsystems auch eine psychologische Betreuung der Betroffenen und der Familien erforderlich. Wesentliches therapeutisches Element, nicht nur im Kindes- und Jugendalter, sondern insbesondere auch im Erwachsenenalter, ist die Kräftigung der Muskulatur durch kontinuierliche Physiotherapie und spezifische Rehabilitationsmaßnahmen. Durch eine Aktivierung der Muskulatur kann ein osteoanaboler Stimulus gesetzt werden und so die Kollagenproduktion angeregt werden. Zusätzlich muss es der Schwerpunkt der physiotherapeutischen Massnahmen sein, die Mobilität und Selbstständigkeit der Betroffenen zu verbessern, um ihnen, in Abhängigkeit von der Schwere der Erkrankung, ein möglichst selbstständiges Leben zu ermöglichen. Hierzu zählt nicht nur die Verbesserung der Steh- und Geh-Fähigkeit auch nach wiederholten Frakturen, sondern auch die multimodale Schmerztherapie. Hier sind ebenfalls ergänzende schmerztherapeutische und psychologische Therapieelemente entscheidend, um den Patientinnen und Patienten zu einem Leben mit einer möglichst uneingeschränkten Teilhabe zu verhelfen [4].

\section{Aktuelle Therapie}

\section{Medikamentöse Therapie}

Für die medikamentöse Therapie der Osteogenesis imperfecta gibt es keine international anerkannten Leitlinien, und die meisten Medikamente können in Deutschland nur im Rahmen eines „individuellen Heilversuchs“ außerhalb der Zulassung der Medikamente eingesetzt werden. Für Erwachsene mit einer OI kann meist über die Zusatz-Diagnose einer Osteoporose eine medikamentöse Therapie begründet werden, da einige Patientinnen und Patienten mit Ol die Diagnosekriterien einer Osteoporose erfüllen.

Während für Erwachsene mehrere therapeutische Optionen bestehen, beschränkt sich die Behandlung im Kindes- und Jugendalter, wenn die Patientinnen und Patienten die intensivsten skelettalen Probleme haben, auf eine antiresorptive Therapie. Die Behandlung mit intravenösen Bisphosphonaten hat sich seit ca. 25 Jahren etabliert und kann, obwohl nicht durch Leitlinien und doppelblinde Studien belegt, als Standardtherapie betrachtet werden, wenn die Betroffenen einen schwereren Verlauf der Erkrankung aufweisen [5]. Zunächst wurde die Behandlung mit dem Bisphosphonat-Wirkstoff Pamidronat durchgeführt [6]. Da dies jedoch mehrfach im Jahr mehrtägige stationäre Aufenthalte im Krankenhaus erfordert, haben sich inzwischen Bisphosphonate durchgesetzt, die ambulant verabreicht werden können. In Deutschland und Italien wird zumeist der Wirkstoff Neridronat im Abstand von drei Monaten intravenös verabreicht, wohingegen im amerikanischen Raum überwiegend Zoledronat genutzt wird $[7,8]$. Bei dem Intervall der Gaben muss der im Kindes- und Jugendalter wesentlich aktivere Knochenstoffwechsel im Vergleich zu Erwachsenen beachtet werden. Der Einsatz von Bisphosphonaten führt zu einer Zunahme der Knochenmasse und einer Abnahme von Knochenschmerzen sowie zu einer Zunahme der Mobilität. [9, 10]. 
Orale Bisphosphonate konnten nur einen geringen Effekt auf die Knochenflächendichte zeigen, ohne dass sich hierdurch die Frakturrate deutlich verändert hat [11]. Daher kommen orale Bisphosphonate derzeit lediglich in seltenen Fällen und bei Personen mit einer leichten Verlaufsform einer Ol in Betracht.

Im Kindes- und Jugendalter laufen derzeit erste Untersuchungen für den Einsatz des Osteoklasten-Antikörpers Denosumab (NCT02352753). Hier zeigt sich bei Verwendung dieses Wirkstoffs eine stärkere Zunahme der Knochenmasse im Kindes- und Jugendalter als während einer Behandlung mit Bisphosphonaten [12]. Allerdings muss der „Rebound“ zum Ende des BehandlungsIntervalls beachtet werden. Hier liegen Berichte über eine Hypercalcämie vor [13]. Diese tritt insbesondere auch am Ende einer hochdosierten Therapie auf. Bisher ist noch kein Behandlungsstandard etabliert, wie eine Behandlung mit Denosumab während der Wachstumsphase beendet werden kann, und sollte daher außerhalb von Studien derzeit nicht verwendet werden

Osteoanabole Medikamente wie z. B. Teriparatid werden derzeit nicht angewendet, da beim wachsenden Skelettsystem im Tierversuch Osteosarkome aufgetreten sind [14]. In Planung sind Studien im Kindes und Jugendalter mit Antisklerostin-Antikörpern, wodurch der Knochenaufbau stimuliert werden soll, bei gleichzeitiger Hemmung des Knochenabbaus.

Im Erwachsenenalter kommen neben den antiresorptiven Therapien mit Bisphosphonaten und Denosumab auch osteoanabole Medikamente, z. B. Teriparatid, zum Einsatz. Der Einsatz von Parathormon wurde in mehreren Studien untersucht und zeigte eine Zunahme der Knochenmasse und eine Verbesserung der chronischen Knochenschmerzen [15]. Eine erste Studie über den Einsatz von Antisklerostin-Antikörpern bei Erwachsenen mit einer leichten Verlaufsform einer OI erbrachte ein gutes Ansprechen der Knochenstoffwechsel-Marker (NCT03118570) [16]. Ergebnisse über die Frakturrate und Knochenflächendichte-Entwicklung bei dieser Studie liegen derzeit noch nicht vor. In Zukunft werden auch Studien zum Einsatz von TGF-beta durchgeführt werden, hierzu liegen aber derzeit nur Erkenntnisse aus dem Tiermodell vor (NCT03064074) [17].

Die medikamentöse Therapie allein ist nicht ausreichend für die Behandlung von Kindern und Erwachsenen mit einer OI. Die Medikamente müssen Teil eines Gesamt-Behandlungs-Konzeptes sein, das alle Bereiche der Betreuung aufeinander abstimmt.

\section{Calcium und Vitamin D}

Die Basisversorgung mit Calcium und Vitamin D bei Ol folgt prinzipiell etablierten osteologischen Gepflogenheiten mit der primären Zielsetzung der Vermeidung einer Mangelsituation und den daraus resultierenden negativen Implikationen für die Skelettmineralisation. Darüber hinaus sollte gerade im unmittelbaren Umfeld einer parenteralen und sehr potenten antiresorptiven Behandlung bei suboptimaler alimentärer Versorgung auch eine Calcium-Supplementation erwogen werden, zur Vermeidung einer in den ersten $48 \mathrm{~h}$ post-infusionem ansonsten durchaus nicht ungewöhnlichen Hypokalzämie [18]. Während ansonsten eine suffiziente Calciumversorgung bei der überwiegenden Mehrzahl der Patientinnen und Patienten alimentär sichergestellt werden kann und sollte, ist für einen suffizienten Vitamin-D-Status in der Mehrzahl der Patientinnen und Patienten eine
Supplementation erforderlich, zumindest während der sonnenschwachen Wintermonate. Die Studienlage hinsichtlich der optimalen Dosierung bzw. anzustrebender Serumspiegel bei Ol ist vergleichsweise überschaubar. Eine vergleichende doppelblinde RCT bei Kindern und Jugendlichen im Alter 6-19 Jahre über ein Jahr zeigt, dass mit 2000 IE täglich im Vergleich zu 400 IE täglich zwar ein signifikant stärkerer Anstieg der Serumspiegel erzielt wird, sich daraus aber zumindest über den Zeitraum von 12 Monaten kein signifikanter Unterschied in der Knochenflächendichte oder der Frakturrate ergibt [19].

\section{Orthopädische/chirurgische Maßnahmen}

Orthopädische Maßnahmen sind ein wichtiger Baustein in der Behandlung von Ol- Patientinnen und Patienten. Wenn eine akute Versorgung im Falle von Frakturen erforderlich wird, muss entschieden werden, ob eine konservative Versorgung (mit Gips oder Schiene) ausreichend ist oder ob operative Massnahmen indiziert sind. Bei ausgeprägten Deformierungen oder dislozierten Frakturen sind in aller Regel operative Maßnahmen erforderlich, die sich naturgemäß im Kindesalter von Maßnahmen bei ausgewachsenen Patientinnen und Patienten unterscheiden.

\section{Behandlung von Kindern und Jugendlichen:}

Bei Patientinnen und Patienten, die mit ausgeprägten Deformierungen geboren werden, ist die motorische Entwicklung oft durch die Verbiegungen der Knochen limitiert. Sobald die Patientinnen und Patienten keine motorischen Fortschritte mehr machen, da es immer wieder zu Schmerzen und Frakturen kommt, sollte eine operative Begradigung geplant werden. Dies ist meist im Alter von ca. 2-3 Jahren der Fall. Es sollte dann mittels Osteotomien eine Korrektur der Deformierung angestrebt werden. Diese werden durch einen intramedullären Nagel stabilisiert. Im Kindes- und Jugendalter sollten hierbei „Teleskopnägel“ verwendet werden. Diese zwei ineinandergeschobenen Nagelanteile werden an den langen Röhrenknochen, überwiegend der unteren Extremität, jeweils jenseits der Wachstumsfugen verankert und können sich während des Wachstums auseinanderziehen (teleskopieren) und so den Knochen während der gesamten Wachstumsphase von innen schienen. Bei dieser technisch aufwendigen Operation können vorhandene Deformierungen korrigiert werden. Die Verwendung solcher Teleskopnägel hat sich inzwischen als Standard etabliert, ist aber dennoch eine technisch schwierige Operation, die nur in spezialisierten Zentren durchgeführt werden sollte [20].

\section{Behandlung von Erwachsenen:}

Die Indikation zur orthopädisch-chirurgischen Intervention im Erwachsenenalter ergibt sich insbesondere bei konservativ nicht sinnvoll therapierbaren Frakturen, bei persistierenden oder erst sekundär aufgetretenen, schwerwiegenden skelettalen Deformierungen sowie bei erkrankungsbedingt häufig vergleichsweise frühzeitig auftretenden arthrotischen Veränderungen. Zwar folgt die Herangehensweise grundsätzlich etablierten chirurgischen Grundprinzipien, die wissenschaftliche Evidenz zu krankheitsspezifischen 


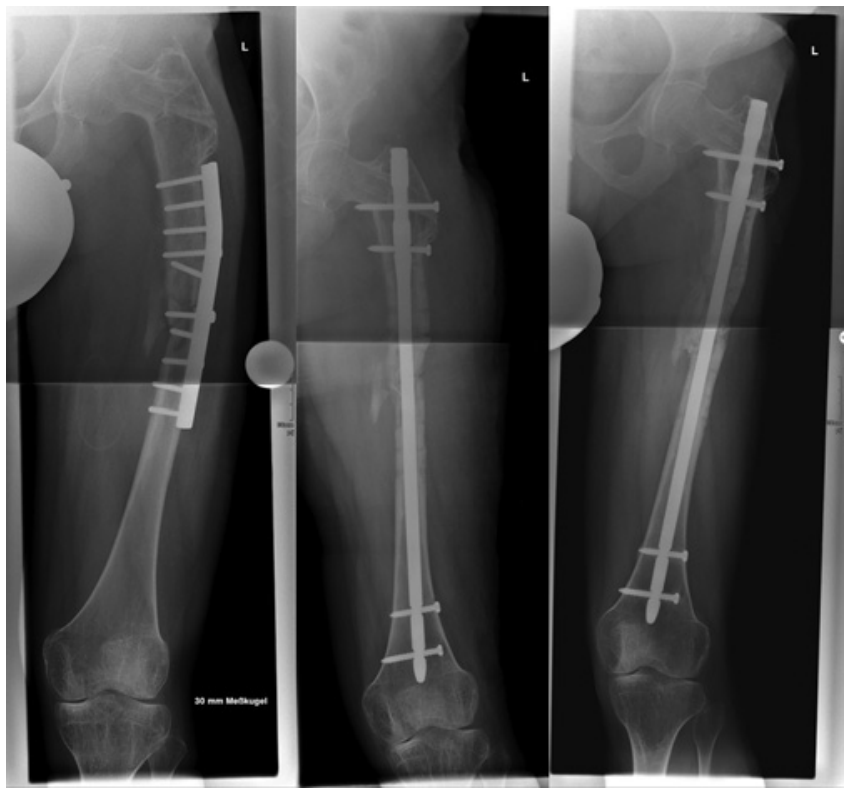

Abb. 1 Plattenosteosynthese mit Pseudarthrose und sekundärem Implantatversagen bei diaphysärer Femurfraktur links. Nach Revision und Marknagelosteosynthese im Verlauf suffiziente Ausheilung.

- Fig. 1 Osteosynthesis with a plate and a resulting pseudarthrosis and secondary diaphyseal femur fracture (left). After revision and insertion of an intramedullary rod sufficient healing of the fracture.

Konzepten ist aber limitiert. Dennoch scheinen einige pathophysiologisch plausible und empirisch etablierte Prinzipien günstig im Hinblick auf eine erfolgreiche Versorgung zu sein. In der Frakturversorgung kann auch bei Ol grundsätzlich von einem suffizienten Heilungspotenzial ausgegangen werden, sodass konservative, funktionell ausgerichtete Ansätze zur Vermeidung der Sekundärkomplikationen aufwendiger chirurgischer Interventionen und langfristiger Immobilisierung durchaus in Betracht gezogen werden sollten und gleichwertige Ergebnisse erbringen können. Bei erforderlicher chirurgischer Intervention sind bei geeigneter Frakturmorphologie und Lokalisation intramedulläre Kraftträger mit langstreckiger Verteilung der auftretenden Kräfte und geringer Hebelwirkung zu bevorzugen ( $\triangleright$ Abb. 1 ).

Gerade bei elektiven chirurgischen Maßnahmen sollte man sich anhand der individuellen Krankheitsgeschichte, Bildgebung, Laborwerte und Knochendichte- und Strukturuntersuchungen einen möglichst umfassenden Überblick über die zu erwartende Knochenqualität, -struktur und -stabilität verschaffen und, sofern zeitlich möglich, auch therapeutische Maßnahmen zu deren Verbesserung initiieren. Grundsätzlich ist es bei guter Vorbereitung durchaus möglich, primär endoprothetische Versorgungen mit Standardverfahren ohne zusätzliche Stabilisierung durchzuführen, d.h. z.B. Hüftendoprothesen ohne Zementverankerung und Knieendoprothesen ohne zementierte Achsverankerung ( Abb. 2). Gerade bei erforderlichen komplexen chirurgischen Eingriffen ist es dabei ganz wesentlich, die medikamentöse Vor- und Nachbehandlung auf das gewählte chirurgische Verfahren abzustimmen ( Abb. 3).

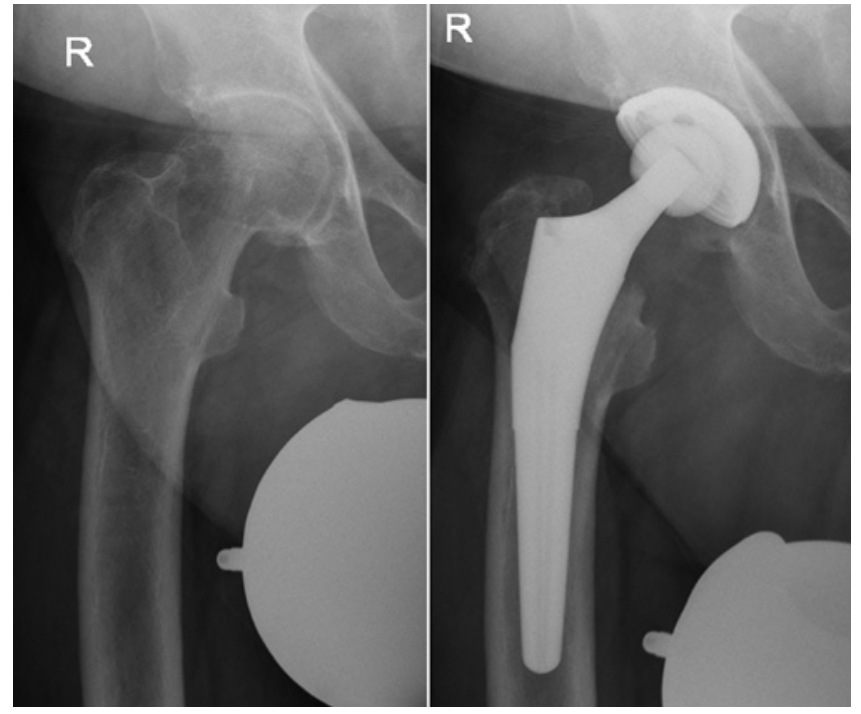

-Abb. 2 Primäre Hüftendoprothese bei immobilisierender Coxarthrose rechtseitig, 60-jähriger Patient mit OI und Adipositas per magna (BMI 50). Stabile zementfreie Versorgung bei suffizienter Vorbehandlung mit Bisphosphonaten.

- Fig. 2 Severe hip arthrosis on the right side in a 60 year old patient with osteogenesis imperfecta and adipositas per magna (BMI $50 \mathrm{~kg} / \mathrm{m}^{2}$ ). Primary hip replacement after previous treatment with bisphosphonates.

\section{Physiotherapie und Rehabilitation}

Die Muskulatur ist ein essenzieller Bestandteil des Skelettsystems und stellt einen der wichtigsten therapeutischen Angriffspunkte bei der Behandlung von Patientinnen und Patienten mit Ol dar. Über die funktionelle Muskel-Knochen-Einheit führt eine Kräftigung der Muskulatur zu einem osteoanabolen Stimulus, der die Synthese von Kollagen durch Osteoblasten anregt. Neben diesem direkten Effekt des Muskeltrainings auf die Knochenmasse müssen aber bei einem physiotherapeutischen Training weitere Aspekte beachtet werden. Regelmäßige Physiotherapie dient zusätzlich dem Erhalt, der Verbesserung und der Wiedererlangung der Gelenkbeweglichkeit. Besonders nach Frakturen und längeren Phasen der Immobilisation, z. B. in einem Gipsverband, muss nicht nur die Muskulatur aufgebaut werden, sondern auch der Bewegungsumfang der Gelenke wieder gesteigert werden.

Zusätzlich zu diesen direkten mechanischen Effekten des Trainings dient die Physiotherapie dazu, komplexe Bewegungsabläufe bei den Patientinnen und Patienten zu verbessern und diese mit den Patientinnen und Patienten zu erlernen. Besonders im Kindes- und Jugendalter erreichen die Betroffenen ihre motorischen Meilensteine meist nicht selbstständig, sondern nur durch ein intensives Training. Durch vorhandene Deformierungen und Dysproportionen ist es für sie schwieriger, sich aufzurichten, das Gleichgewicht zu halten und sich fortzubewegen. Hier dient die Physiotherapie dazu, diese Bewegungsabläufe in einem sicheren Umfeld zu erlernen.

Bei Erwachsenen stehen der Erhalt der motorischen Funktionen sowie eine Reduktion chronischer Schmerzen im 

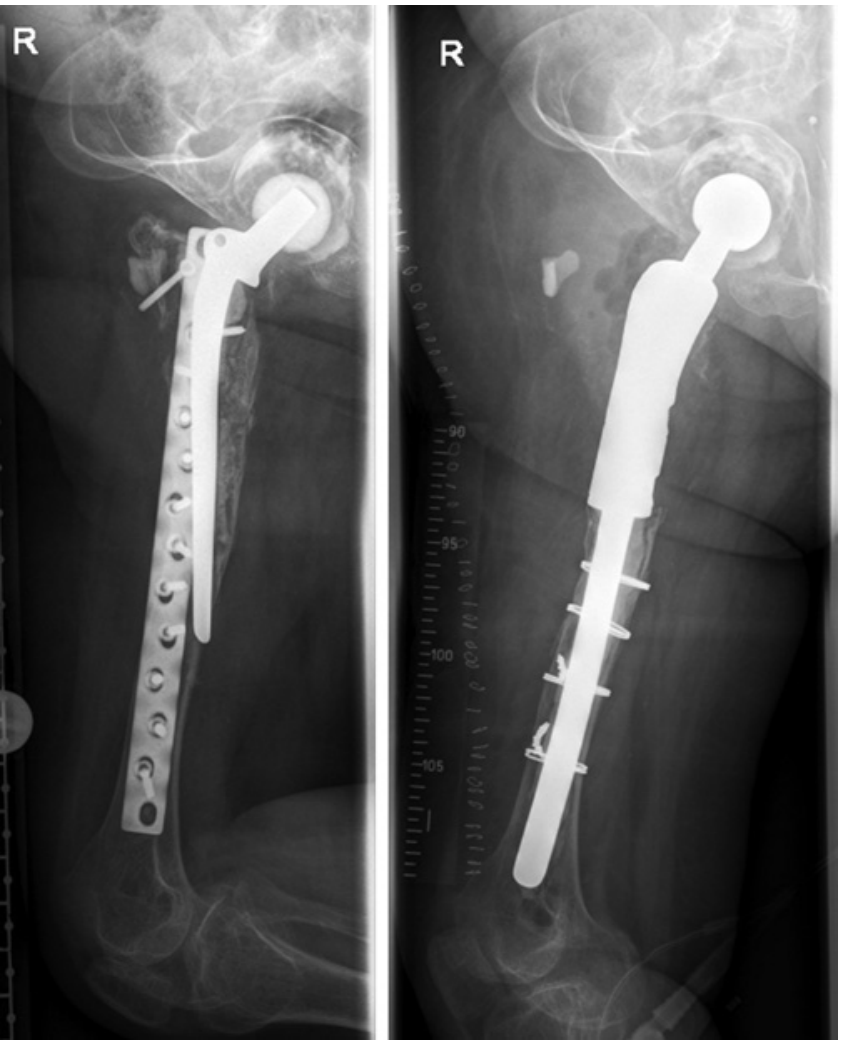

-Abb. 3 Komplexe Revisionssituation bei ausgebrochenem Femurschaft und einliegender Plattenosteosynthese nach älterer periprothetischer Fraktur. Chirurgische Versorgung im Intervall nach Optimierung der Basisversorgung mit Vitamin D und Erweiterung der knochenspezifischen Therapie um eine osteoanabole Komponente.

- Fig. 3 Difficult situation on the right femur with long plate after femur fracture. After supplementation with Calcium and Vitamin D surgical intervention and treatment with osteoanabolic medication.

Vordergrund. Bei vielen Patientinnen und Patienten kommt es im Laufe des Lebens durch die vorhandenen Fehlstellungen zu einer verstärkten Gelenkabnutzung mit resultierenden Schmerzen und einer Abnahme der Mobilität. Neben regelmäßiger Physiotherapie sind bei diesen Patientinnen und Patienten oft rehabilitative Maßnahmen sinnvoll, um die Selbstständigkeit im Alltag und die Erwerbsfähigkeit zu erhalten. Spezielle physiotherapeutische Konzepte für Ol sind international bisher nicht etabliert. Erste Versuche einer Konsensusfindung wurden im Kindes- und Jugendalter durchgeführt [21]. Für Erwachsene orientiert sich die Physiotherapie meist an den Empfehlungen der Behandlung von Patientinnen und Patienten mit Osteoporose sowie an dem Ziel einer allgemeinen Kräftigung der Muskulatur. Zur Schmerztherapie können sowohl Massagen als auch gezielte Kräftigungsübungen angewendet werden.

\section{Genetik}

Während bei der OI klinisch die erhöhte Knochenfragilität im Mittelpunkt steht, wird diese Erkrankungsgruppe genetisch und pathophysiologisch zuerst mit der Produktion und Modifikation von Typ-1-Kollagen assoziiert [22]. In den weitaus meisten Fällen finden sich Mutationen in den Genen für die alpha-1- und alpha2-Ketten des Typ-1-Kollagens, COL1A1 und COL1A2. Hierbei sind generell Mutationen, die zu einem verfrühten Stopcodon führen, milder als die klassischen Glycin-Mutationen, die die Bildung der Kollagen-Tripelhelix verhindern. Außerdem sind die Verläufe bei COL1A2-Mutationen im Durchschnitt gutartiger als bei COL1A1 [23]. Wurden von beiden Genen erfolgreich mRNAs erzeugt und an Ribosomen translatiert, müssen die Polypeptidkette modifiziert und die Tripelhelix gebildet werden. Hierbei helfen verschiedene Proteine, in denen genetische Defekte folglich ebenfalls eine Ol erzeugen können. Hier ist zum einen die Prolylhydroxylase zu nennen, ein Komplex aus den Proteinen Cyclophilin B (Gen PPIB), CRTAP und P3H1 (Genname wird aufgeführt, wenn nicht mit Proteinname identisch) [24]. Andererseits sind an Faltung und intrazellulärem Transport die Chaperone FKBP10 und HSP47 (Gen SERPINH1) beteiligt. Anschließend erfolgt die Hydroxylierung von Lysinresten durch PLOD2, bevor schließlich nach der Sekretion der Tripelhelix die N- und C-terminalen Propeptide durch die Protease BMP1 abgespalten werden, damit Fibrillen und Fasern gebildet werden können.

Von den oben genannten Gendefekten haben nur die in den Genen COL1A1 und COL1A2 eine dominante Wirkung, d. h., eine Mutation eines Allels reicht bereits aus, um die Erkrankung auszulösen. Dies erklärt auch, warum diese Art der OI am häufigsten anzutreffen ist. Bei diesen Fällen kann eine positive Familienanamnese vorliegen. Die Abwesenheit von weiteren Betroffenen in der Familie schließt die Diagnose aber nicht aus, da Neumutationen auftreten [25]. Alle anderen genannten Gene/Genprodukte sind mit autosomal-rezessiver Vererbung verbunden. Sie sind sehr viel seltener, da hier von beiden Elternteilen je eine Mutation in dem jeweiligen Gen weitergegeben werden muss. Das Risiko hierfür ist größer bei Blutsverwandtschaft der Eltern. Die einzige X-chromosomale Form der Ol wird durch Mutationen im Gen MBTPS2 verursacht, das eine Protease im Golgi-Kompartiment produziert [26]. Hier sind nur Männer betroffen, Frauen sind nicht betroffene Überträgerinnen. Ein weiteres Gen, das mit einer dominanten OI verknüpft ist, ist IFITM5 [27]. Die hierdurch verursachte OI Typ $\mathrm{V}$ ist aber viel seltener als die durch COL1A1 und COL1A2 verursachten Formen, da nur zwei bestimmte Veränderungen in dem Gen den Phänotyp auslösen. Die Funktion von IFITM5 ist nicht vollständig verstanden, hat aber nicht direkt mit Typ-1-Kollagen zu tun. Nimmt man also einen Bezug zu Typ-1-Kollagen als Grundlage der Krankheitsdefinition, wäre OI Typ V keine OI, was klinisch natürlich nicht sinnvoll wäre. Andererseits verursachen Defekte von PLOD2 und FKBP10 das Bruck-Syndrom, eine Erkrankung mit Frakturen und Kontrakturen [28]. Obwohl beide direkt mit Typ-1-Kollagen interagieren, werden sie oft nicht zur OI gezählt. Hier gibt es also Inkonsistenzen, die möglicherweise durch eine überdachte Nosologie unter Einbeziehung genetischer, pathophysiologischer und - in immer größerem Maße - therapeutischer Aspekte ausgeräumt werden könnten [29]. Ein Überblick über die verschiedenen Formen ist in > Tab. 1 dargestellt. 
-Tab.1. Übersicht über die für eine Osteogenesis imperfecta ursächlichen Gene mit Vererbungsmodus und entsprechendem OITyp.

- Table 1 Disease causing genes, way of inheritance and clinical OI type.Table 1:

\begin{tabular}{|c|c|c|c|}
\hline Gen & $\begin{array}{l}\text { Verer- } \\
\text { bung }\end{array}$ & OI-Typ & Funktion \\
\hline COL1A1 & $A D$ & $\begin{array}{l}\text { Typ I, II, } \\
\text { III, IV }\end{array}$ & EZM \\
\hline COL1A2 & $A D$ & $\begin{array}{l}\text { Typ II, III, } \\
\text { IV }\end{array}$ & EZM \\
\hline IFITM5 & $A D$ & Typ V & $\begin{array}{l}\text { Parakrine } \\
\text { Regulation? }\end{array}$ \\
\hline BMP1 & AR & Typ XIII & Spaltung Propeptide \\
\hline CREB3L1 & AR & Typ XVI & ER-Funktion \\
\hline CRTAP & $A R$ & Typ VII & $\begin{array}{l}\text { Prolyl- } \\
\text { Hydroxylierung }\end{array}$ \\
\hline FKBP10 & $A R$ & $\begin{array}{l}\text { Typ XI, } \\
\text { Bruck- } \\
\text { Syndrom }\end{array}$ & Faltung, Transport \\
\hline MESD & $A R$ & Typ XX & Wnt-Signalweg \\
\hline P3H1 & AR & Typ VIII & $\begin{array}{l}\text { Prolyl- } \\
\text { Hydroxylierung }\end{array}$ \\
\hline PLOD1 & AR & $\begin{array}{l}\text { Bruck- } \\
\text { Syndrom }\end{array}$ & Lysyl-Hydroxylierung \\
\hline PPIB & $A R$ & Typ IX & $\begin{array}{l}\text { Prolyl- } \\
\text { Hydroxylierung }\end{array}$ \\
\hline SERPINF1 & AR & Typ VI & Parakrine Regulation \\
\hline SERPINH1 & $A R$ & Typ X & Faltung, Transport \\
\hline SP7 & AR & Typ XII & OB-Differenzierung \\
\hline SPARC & $A R$ & Typ XVII & EZM \\
\hline TENT5A & $A R$ & Typ XVIII & unbekannt \\
\hline TMEM38B & AR & Typ XIV & Sekretion \\
\hline WNT1 & $A R$ & Typ XV & Wnt-Signalweg \\
\hline MBTPS2 & $X L R$ & Typ XIX & Sekretion \\
\hline
\end{tabular}

\section{Besondere Formen}

In den letzten Jahren konnten die molekulargenetischen Ursachen für einige seltene Formen der OI identifiziert werden. Zwei dieser Sonderformen werden im Folgenden ausführlicher dargestellt, da die Kenntnis dieser Formen zukünftig therapeutische Konsequenzen haben könnte.

\section{Osteogenesis imperfecta, verursacht durch Mutationen in SERPINF1}

Osteogenesis imperfecta Typ VI gehört zu den seltenen autosomal-rezessiven Ol-Formen. Diese Form war schon lange durch ihren besonderen Phänotyp charakterisiert worden, ohne dass hierfür die molekulare Grundlage bekannt war [30]. Die Patientinnen und Patienten zeigen bei Geburt nur geringe skelettale Symptome. Die Frakturen beginnen meist im zweiten Lebensjahr. $\mathrm{Ab}$ dann sind Frakturen und auch Deformierungen progredient.
Die Betroffenen weisen keine anderen Anzeichen einer Kollagenopathie auf. Die Skleren und Zähne sind nicht betroffen, die Muskulatur ist häufig leicht hypoton, jedoch ohne Hypermobilität der Gelenke [30]. Besonders das schlechte Ansprechen des Skelettsystems auf eine Behandlung mit Bisphosphonaten legte die Vermutung einer anderen Pathophysiologie nahe. Der Nachweis ursächlicher Mutationen im Gen SERPINF1 bewies dann die veränderte Pathophysiologie, die bei diesen Patientinnen und Patienten zur Instabilität des Skelettsystems führt [31]. SEPINF1 kodiert für das Protein „Pigment epithelium-derived factor (PEDF)“. Ein Funktionsverlust von SERPINF1 führt zu einer vermehrten Differenzierung und Aktivierung von Osteoklasten, vermittelt über das RANKL/Osteoprotegerin-System.

In primären Osteoblasten wurde nachgewiesen, dass die Expression von OPG durch den von SERPINF1 kodierten Faktor PEDF (pigment epithelium derived factor) reguliert wird und so die Aktivität von Osteoklasten beeinflusst wird [32]. PEDF beeinflusst zusätzlich die Differenzierung von mesenchymalen Stammzellen sowie die Mineralisierung über den ERK und AKTSignalweg [33].

Eine durch Mutationen in SERPINF1 reduzierte Konzentration von PEDF bewirkt eine vermehrte Aktivität von Osteoklasten über den RANKL/RANK/NFKB-Signalweg und somit einen vermehrten Knochenabbau bei den Patientinnen und Patienten, beginnend nach der Geburt.

Bei Patientinnen und Patienten mit dieser Sonderform einer Ol wurde erstmalig der Osteoklasten-Antikörper Denosumab angewendet. Die Behandlung führte zu einer irreversiblen Hemmung der Osteoklasten, die sich bei Patientinnen und Patienten mit OI VI als effektiver herausgestellt hat als eine Behandlung mit Bisphosphonaten. Bisher sind allerdings die richtigen Dosierungsintervalle noch nicht gefunden und die Frage, wie ein „Rebound“ nach Ende der Therapie verhindert werden kann, noch nicht gelöst. Dennoch waren die Ergebnisse bei den Patientinnen und Patienten mit Mutationen in SERPINF1 und im „oim/oim Mausmodell" so positiv, dass derzeit auch eine klinische Studie zur Wirksamkeit von Denosumab bei Kindern mit einer klassischen Ol, verursacht durch Mutationen in COL1A1/2, untersucht wird (NCT02352753).

\section{Osteogenesis imperfecta, verursacht durch Mutationen in WNT1}

Osteogenesis imperfecta Typ XV gehört zu den sehr seltenen autosomal-rezessiven OI-Formen und wird durch homozygote oder compound-heterozygote Mutationen im WNT1-Gen verursacht. Anders als bei den autosomal-dominant vererbten, klassischen OlFormen liegt keine Störung eines Knochenstrukturproteins (Kollagen Typ I), sondern die eines zentralen Regulators des Knochenstoffwechsels vor. Diese zentrale Rolle von WNT1 wird dadurch unterstrichen, dass auch heterozygote Mutationen in diesem Gen zu einem Knochenphänotyp, nämlich zu einer hereditären EarlyOnset-Osteoporose (EOOP) führen, die durch einen ausgeprägten Low-turnover-Knochenstoffwechsel gekennzeichnet ist [34]. Kinder mit OI Typ XV sind schwer betroffen und weisen bereits im jüngsten Lebensalter viele Frakturen und Deformierungen der langen Röhrenknochen auf. Die Skleren sind weiß, und eine Hirnbeteiligung im Sinne einer geistigen Retardierung ist möglich (s. ^ Abb. 4). 


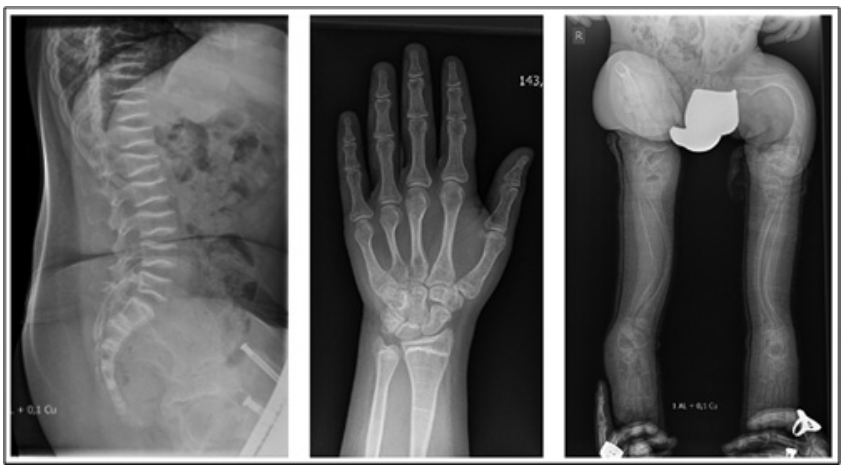

-Abb. 4 Klinische Präsentation von Patientinnen und Patienten mit OI Typ XV. Links - Röntgen Wirbelsäule seitlich mit dem Nachweis multipler Wirbelkörperfrakturen im Alter von 15 Jahren trotz i. v. Bisphosphonat-Therapie seit dem 3. Lebensjahr. Mitte - Röntgen Handgelenk ap desselben Patienten mit Darstellung der typischen Zebralinien als Folge der i.v. Bisphosphonat-Therapie. Rechts Röntgen der unteren Extremitäten eines schwer betroffenen Patienten im Alter von 3 Jahren mit OI Typ XV, geistiger Retardierung und Unfähigkeit zum Sitzen, Gehen und Stehen. Dargestellt sind die auffälligen Verformungen der langen Röhrenknochen der Oberund Unterschenkel.

- Fig. 4 Patient with OI type XV. Left side: $x$-ray of the lateral spine at 15 years of age with multiple vertebral fractures despite bisphosphonate treatment since 3 years of age. Middle: hand $x$-ray of the same patient showing typical 'zebra lines' following i. v. bisphosphonate treatment. Right: Severe deformities of upper and lower legs in a patient with a severe OI type $\mathrm{XV}$, mental retardation an impaired mobility (not able to sit independently).

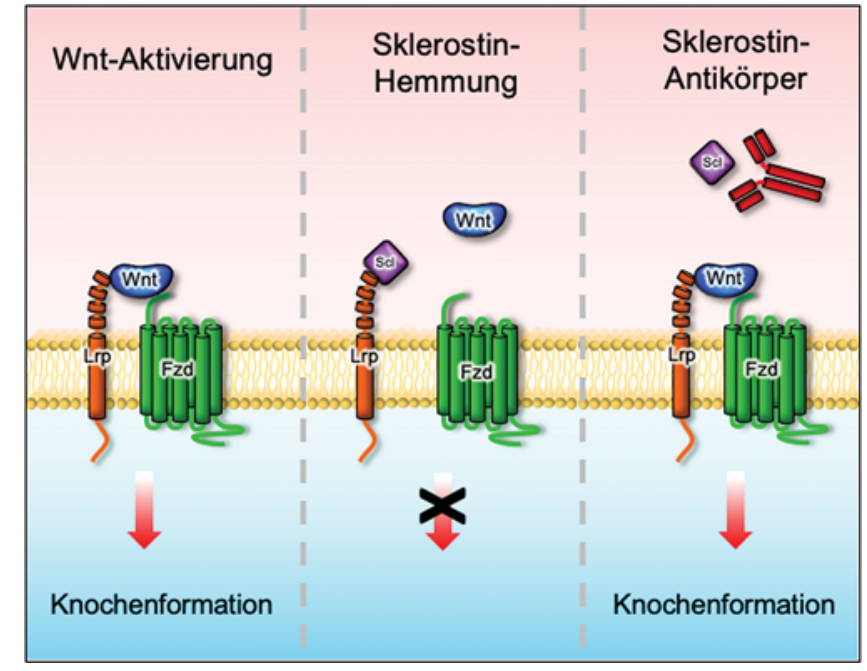

-Abb. 5 Vereinfachte schematische Darstellung des WNT-Signalwegs nach Yorgan et al. (2014). Links - Physiologische Aktivierung der Knochenformation über Aktivierung des WNT-Signalweges im Osteoblasten über LRP- und Fzd-Rezeptoren. Mitte - Hemmung der WNT-Aktivierung durch Sklerostin. Rechts - Aktivierung des WNT-Signalweges bei aufgehobener Sklerostin-Hemmung durch Sklerostin-Antikörper.

-Fig. 5 Schematic presentation of Wnt signaling adapted from Yorgan et al 2014. Left - Activation of Wnt signaling by binding of a Wnt molecule to Fzd receptor and Lrp co-receptor. Middle - Inhibition of Wnt signaling by binding of Sclerostin to Lrp co-receptor. Right - Neutralization of Sclerostin by Sclerostin antibody enables physiological activation of Wnt signaling.
Der Name Wnt leitet sich von wingless - flügellos -, dem offensichtlichen Phänotyp von Fruchtfliegen (Drosophila melanogaster) mit Mutationen in diesem Gen [35], und Int1 - Integrationsstelle 1 - ab, aufgrund der häufigen Integration des Genoms von murinen Mammatumorviren in diesen Bereich [36]. Es gibt 19 verschiedene Wnt-Liganden [37], die mit verschiedenen Rezeptoren interagieren können. Nachdem Wnt1 im Mausmodell zunächst als wesentlicher Faktor der Hirnentwicklung identifiziert wurde [38, 39], wurde dessen Bedeutung für den Knochenstoffwechsel erkannt. Dies gelang u.a. durch den Nachweis einer erhöhten Frakturneigung und eines Knochenmasseverlustes bei partiellem Funktionsverlust oder Zelltyp-spezifischer Inaktivierung sowie eines ausgeprägten, LRP5-unabhängigen, osteoanabolen Effektes bei Aktivierung von WNT1 [40, 41]. Generell scheint die Wirkung von Wnt1 im Skelettsystem primär über eine Modulation der Osteoblasten-Aktivität zu erfolgen.

Die durch Wnt induzierten intrazellulären Signalkaskaden werden in den sogenannten kanonischen und nichtkanonischen Signalwege unterschieden. Diese sind hochkomplex reguliert und bis heute nicht vollständig verstanden, da in den meisten Fällen die exakte Kombination aus Ligand und Rezeptor noch nicht etabliert werden konnte [37].

Zu den eigentlichen Wnt-Rezeptoren gehören Frizzled-Proteine, von denen 10 verschiedene bekannt sind [42] und deren Interaktion mit Wnt teilweise von LRP5- und LRP6-Korezeptoren abhängig ist [43]. Darüber hinaus gibt es noch alternative Bindungsproteine und diverse extrazelluläre Moleküle, die in diese Interaktionen eingreifen [44]. Eines dieser extrazellulären Moleküle ist Sklerostin, ein potenter Inhibitor der Knochenformation. Dessen Entdeckung gelang durch den Nachweis inaktivierender Mutationen im SOSTGen als molekulargenetische Ursache der autosomal-rezessiven sklerosierenden Knochendysplasie und der Van-Buchem-Erkrankung [45]. Das Protein des SOST-Gens wurde folgerichtig Sklerostin genannt und avancierte unmittelbar zu einem Zielprotein für die Entwicklung neuer Therapiestrategien von systemischen Knochenmasseverlust-Syndromen wie der Osteoporose. Es konnte im Folgenden gezeigt werden, dass Anti-Sklerostin-Antikörper tatsächlich den hemmenden Einfluss von Sklerostin auf den Wnt-Signalweg reduzieren können [46]. Dieses Konzept wurde weiterentwickelt und mittlerweile erfolgreich im Menschen angewendet [47], sodass seit diesem Jahr mit Romosozumab, ein Anti-Sklerostin-Antikörper, für die schwere, postmenopausale Osteoporose zugelassen werden konnte (siehe - Abb. 5).

Im Rahmen einer Phase-2a-Studie wurde mit einem anderen Sklerostin-Antikörper (BPS804) auch bereits der proof-of-concept für dieses Wirkprinzip bei Osteogenesis Imperfecta erbracht [16], und derzeit laufen Studien zum weitergehenden Einsatz dieser Substanz (Setrusumab) bei Osteogenesis Imperfecta.

Aufgrund der oben dargestellten Zusammenhänge, insbesondere der reduzierten Osteoblasten-Aktivität, ist es pathophysiologisch sinnvoll, bei systemischen Knochenerkrankungen wie der OI Typ XV oder der WNT1-abhängigen EOOP initial 
osteoanabole Therapiekonzepte zu favorisieren. So konnte z. B. bereits gezeigt werden, dass eine Therapie mit Teriparatid (Forsteo ${ }^{\circledR}$ ) bei WNT1-abhängiger EOOP wirksam ist [41]. Dieser Nachweis steht für Romosozumab allerdings noch aus. Ein großes klinisches Problem sind aber vor allem das begrenzte Indikationsspektrum der erwähnten Substanzen sowie der sehr begrenzte Anwendungszeitraum. Studien zur Anwendung bei Kindern fehlen bisher ganz bzw. verbietet sich die Anwendung von Teriparatid im wachsenden Skelett (s.o.). Daher ist es dringend erforderlich, diese neuen spezifischen Wirkstoffe systematisch im Kontext von OI Typ XV und verwandten Erkrankungen zu untersuchen, um den Patientinnen und Patienten zukünftig gezielte Therapien anbieten zu können, die über eine überwiegend symptomatische Behandlung hinausgehen.

\section{Schlussfolgerung}

Die Osteogenesis imperfecta ist eine seltene genetische Erkrankung mit einer erhöhten Fragilität des Skelettsystems. Durch fortschreitende Kenntnisse über die heterogenen genetischen Ursachen wandelt sich die früher klar definierte Erkrankung zu einem Symptomkomplex mit erhöhter Frakturneigung, Knochenschmerzen und variablen extraskelettalen Symptomen. Bei den meisten Betroffenen liegen Mutationen in den Genen COL1A1/A2 vor und führen zu einer quantitativen (meist klinisch leichtere Verlaufsformen) oder qualitativen (meist schwerere Verlaufsformen) Kollagensynthesestörung. Rezessive Formen sind selten und können auf allen Stufen der Kollagenmodifikation oder des Knochenstoffwechsels zu einer erhöhten Frakturneigung führen. Das sich verbessernde pathophysiologische Verständnis eröffnet die Chance, die Therapie zielgerichteter durchzuführen. Derzeit beruht die Therapie auf einem multimodalen Ansatz, der medikamentöse Therapien, orthopädisch/chirurgische Maßnahmen und Physiotherapie/Rehabilitation beinhaltet. Es bestehen altersabhängige Unterschiede bei der medikamentösen Therapie und bei den operativen Maßnahmen. Während des Körperlängenwachstums, wenn die meisten Frakturen auftreten, sind ausschließlich antiresorptive Therapien untersucht, während bei Erwachsenen auch osteoanabole Medikamente angewendet werden. Das Einbringen von Teleskop-Nägeln beim wachsenden Skelettsystem ist, obwohl technisch anspruchsvoll, als Standard bei schwer betroffenen Patientinnen und Patienten anzusehen. Insgesamt müssen alle Therapien aufeinander abgestimmt sein und das gemeinsame Ziel der größtmöglichen Selbstständigkeit und Teilhabe im Alltag haben.

\section{Funding Information}

Teile dieser Arbeit wurden erstellt im Rahmen der DFG-Forschergruppe FOR 2722 und wurden unterstützt von der „LeppingStiftung“

Die Open Access Publikation dieses Artikels wurde von den Firmen Alexion, Kyowa Kirin und Takeda durch die Übernahme der APC ermöglicht. Es erfolgte keinerlei Einflussnahme dieser Firmen auf die Inhalte des Artikels.

\section{Interessenkonflikt}

Die Autoren erklären, dass keine Interessenkonflikte im Hinblick auf die hier behandelte Thematik bestehen.

OS hat in den vergangenen 3 Jahren in Bezug zur in diesem Artikel behandelten Thematik an klinischen Studien der Firma Amgen teilgenommen und Beratungstätigkeiten für die Firma Mereo durchgeführt sowie Forschungsunterstützung der Stiftung „Care4Brittle Bones“ erhalten.

UK gibt an, keinen Interessenkonflikt zu haben.

RO gibt an, keinen Interessenkonflikt zu haben.

LS erhielt in den vergangenen 3 Jahren Honorar für Vorträge und Beratungstätigkeit von Lilly, Amgen und UCB.

\section{Literatur}

[1] Marini JC, Forlino A, Bächinger HP et al. Osteogenesis imperfecta. Nat Rev Dis Primers 2017; 3: 17052. doi: 10.1038/nrdp.2017.52

[2] Rauch F, Glorieux FH. Osteogenesis imperfecta. Lancet 2004; 363 (9418): 1377-1385

[3] Kashii M, Kanayama S, Kitaoka T et al. Development of scoliosis in young children with osteogenesis imperfecta undergoing intravenous bisphosphonate therapy. J Bone Miner Metab 2019; 37 (3): 545-553. doi: 10.1007/s00774-018-0952-x

[4] Hoyer-Kuhn H, Rehberg M, Semler O. Angeborene Skeletterkrankungen. Monatsschrift Kinderheilkunde 2017; 165 (8): 663-671

[5] Land C, Rauch F, Montpetit K et al. Effect of intravenous pamidronate therapy on functional abilities and level of ambulation in children with osteogenesis imperfecta. J Pediatr 2006; 148 (4): 456-460. doi: 10.1016/j.jpeds.2005.10.041

[6] Glorieux FH, Bishop NJ, Plotkin H et al. Cyclic administration of pamidronate in children with severe osteogenesis imperfecta. N Engl J Med 1998; 339 (14): 947-952. doi: 10.1056/NEJM199810013391402

[7] Gatti D, Antoniazzi F, Prizzi R et al. Intravenous neridronate in children with osteogenesis imperfecta: a randomized controlled study. J Bone Miner Res 2005; 20 (5): 758-763. doi: 10.1359/JBMR.041232

[8] Kumar C, Panigrahi I, Somasekhara Aradhya A et al. Zoledronate for Osteogenesis imperfecta: evaluation of safety profile in children. J Pediatr Endocrinol Metab 2016; 29 (8): 947-952. doi: 10.1515/jpem2015-0351

[9] Land C, Rauch F, Munns CF et al. Vertebral morphometry in children and adolescents with osteogenesis imperfecta: effect of intravenous pamidronate treatment. Bone 2006; 39 (4): 901-906. doi: 10.1016/j. bone.2006.04.004

[10] Hoyer-Kuhn H, Bartz-Seel J, Blickheuser R et al. Diagnostik und Therapie der Osteogenesis imperfecta. Monatsschrift Kinderheilkunde 2016; 165 (4): 333-346. doi: 10.1007/s00112-016-0189-5

[11] Bishop N, Adami S, Ahmed SF et al. Risedronate in children with osteogenesis imperfecta: a randomised, double-blind, placebo-controlled trial. Lancet 2013; 382 (9902): 1424-1432. doi: 10.1016/S0140-6736 (13)61091-0

[12] Hoyer-Kuhn H, Franklin J, Allo G et al. Safety and efficacy of denosumab in children with osteogenesis imperfect-a first prospective trial. J Musculoskelet Neuronal Interact 2016; 16 (1): 24-32

[13] Trejo P, Rauch F, Ward L. Hypercalcemia and hypercalciuria during denosumab treatment in children with osteogenesis imperfecta type VI. J Musculoskelet Neuronal Interact 2018; 18 (1): 76-80

[14] Vahle JL, Sato M, Long GG et al. Skeletal changes in rats given daily subcutaneous injections of recombinant human parathyroid hormone (1-34) for 2 years and relevance to human safety. Toxicol Pathol 2002; 30 (3): 312-321. doi: 10.1080/01926230252929882 
[15] Orwoll ES, Shapiro J, Veith S et al. Evaluation of teriparatide treatment in adults with osteogenesis imperfecta. J Clin Invest 2014; 124 (2): 491-498. doi: 10.1172/JCI71101

[16] Glorieux FH, Devogelaer JP, Durigova M et al. BPS804 anti-sclerostin antibody in adults with moderate osteogenesis imperfecta: results of a randomized phase 2a trial. J Bone Miner Res 2017; 32 (7): 1496-1504. doi: $10.1002 / j b m r .3143$

[17] Tauer JT, Abdullah S, Rauch F. Effect of anti-TGF-beta treatment in a mouse model of severe osteogenesis imperfecta. J Bone Miner Res 2019; 34 (2): 207-214

[18] Maines E, Tadiotto E, Morandi $G$ et al. Hypocalcemia following neridronate administration in pediatric patients with osteogenesis imperfecta: a prospective observational study. J Pediatr Genet 2020; 9 (2): 93-100. doi: 10.1055/s-0039-1700972

[19] Plante L, Veilleux LN, Glorieux FH et al. Effect of high-dose vitamin D supplementation on bone density in youth with osteogenesis imperfecta: a randomized controlled trial. Bone 2016; 86: 36-42. doi: 10.1016/j.bone.2016.02.013

[20] Wirth T. Osteogenesis imperfecta. Orthopade. 2012; 41 (9): 773-782; quiz 83-4.

[21] Mueller B, Engelbert R, Baratta-Ziska F et al. Consensus statement on physical rehabilitation in children and adolescents with osteogenesis imperfecta. Orphanet J Rare Dis 2018; 13 (1): 158. doi: 10.1186/ s13023-018-0905-4

[22] Forlino A, Marini JC. Osteogenesis imperfecta. Lancet 2016; 387 (10028): 1657-1671

[23] Pollitt R, McMahon R, Nunn J et al. Mutation analysis of COL1A1 and COL1A2 in patients diagnosed with osteogenesis imperfecta type I-IV. Hum Mutat 2006; 27 (7): 716. doi: 10.1002/humu.9430

[24] Van Dijk FS, Sillence DO. Osteogenesis imperfecta: clinical diagnosis, nomenclature and severity assessment. Am J Med Genet A 2014; 164A (6): $1470-1481$

[25] Mrosk J, Bhavani GS, Shah Het al. Diagnostic strategies and genotypephenotype correlation in a large Indian cohort of osteogenesis imperfecta. Bone 2018; 110: 368-377. doi: 10.1016/j.bone.2018.02.029

[26] Lindert U, Cabral WA, Ausavarat $S$ et al. MBTPS2 mutations cause defective regulated intramembrane proteolysis in X-linked osteogenesis imperfecta. Nat Commun 2016; 7: 11920. doi:10.1038/ncomms 11920

[27] Semler O, Garbes L, Keupp K et al. A mutation in the 5'-UTR of IFITM5 creates an in-frame start codon and causes autosomal-dominant osteogenesis imperfecta type $\mathrm{V}$ with hyperplastic callus. Am J Hum Genet 2012; 91 (2): 349-357. doi: 10.1016/j.ajhg.2012.06.011

[28] Breslau-Siderius E], Engelbert RH, Pals G et al. Bruck syndrome: a rare combination of bone fragility and multiple congenital joint contractures. J Pediatr Orthop B 1998; 7 (1): 35-38

[29] Tauer JT, Robinson ME, Rauch F. Osteogenesis imperfecta: new perspectives from clinical and translational research. JBMR Plus 2019; 3 (8): e10174

[30] Glorieux FH, Ward LM, Rauch F et al. Osteogenesis imperfecta type VI: a form of brittle bone disease with a mineralization defect. J Bone Miner Res 2002; 17 (1): 30-38. doi: 10.1359/jbmr.2002.17.1.30
[31] Becker J, Semler O, Gilissen C et al. Exome sequencing identifies truncating mutations in human SERPINF1 in autosomal-recessive osteogenesis imperfecta. Am J Hum Genet 2011; 88 (3): 362-371. doi:10.1016/j. ajhg.2011.01.015

[32] Akiyama T, Dass CR, Shinoda Yet al. PEDF regulates osteoclasts via osteoprotegerin and RANKL. Biochem Biophys Res Commun 2010; 391 (1): 789-794. doi: 10.1016/j.bbrc.2009.11.139

[33] Li F, Na S, Tombran-Tink J et al. Pigment epithelium-derived factor enhances differentiation and mineral deposition of human mesenchymal stem cells. Stem Cells 2013; 31 (12): 2714-2723

[34] Keupp K, Beleggia F, Kayserili H et al. Mutations in WNT1 cause different forms of bone fragility. Am J Hum Genet 2013; 92 (4): 565-574. doi: 10.1016/j.ajhg.2013.02.010

[35] Nusslein-Volhard C, Wieschaus E. Mutations affecting segment number and polarity in Drosophila. Nature 1980; 287 (5785): 795-801

[36] Nusse R, Varmus HE. Many tumors induced by the mouse mammary tumor virus contain a provirus integrated in the same region of the host genome. Cell 1982; 31 (1): 99-109

[37] Saito-Diaz K, Chen TW, Wang X et al. The way Wnt works: components and mechanism. Growth Factors 2013; 31 (1): 1-31. doi: 10.3109/ 08977194.2012.752737

[38] McMahon AP, Bradley A. The Wnt-1 (int-1) proto-oncogene is required for development of a large region of the mouse brain. Cell 1990; 62 (6): 1073-1085

[39] Thomas KR, Capecchi MR. Targeted disruption of the murine int-1 proto-oncogene resulting in severe abnormalities in midbrain and cerebellar development. Nature 1990; 346 (6287): 847-850

[40] Joeng KS, Lee YC, Jiang MM et al. The swaying mouse as a model of osteogenesis imperfecta caused by WNT1 mutations. Hum Mol Genet 2014; 23 (15): 4035-4042. doi: 10.1093/hmg/ddu117

[41] Luther J, Yorgan TA, Rolvien T et al. Wnt1 is an Lrp5-independent boneanabolic Wnt ligand. Sci Transl Med 2018; 10: 466. doi:10.1126/scitransImed.aau7137

[42] Clevers H, Nusse R. Wnt/beta-catenin signaling and disease. Cell 2012; 149 (6): 1192-1205

[43] Tamai K, Semenov M, Kato Y et al. LDL-receptor-related proteins in Wnt signal transduction. Nature 2000; 407 (6803): 530-535. doi:10.1038/ 35035117

[44] Bovolenta P, Esteve P, Ruiz JM et al. Beyond Wnt inhibition: new functions of secreted frizzled-related proteins in development and disease. J Cell Sci 2008; 121 (Pt 6): 737-746. doi: 10.1242/jcs.026096

[45] Balemans W, Patel N, Ebeling Met al. Identification of a $52 \mathrm{~kb}$ deletion downstream of the SOST gene in patients with van Buchem disease. J Med Genet 2002; 39 (2): 91-97. doi: 10.1136/jmg.39.2.91

[46] van Dinther M, Zhang J, Weidauer SE et al. Anti-Sclerostin antibody inhibits internalization of Sclerostin and Sclerostin-mediated antagonism of Wnt/LRP6 signaling. PLoS One 2013; 8 (4): e62295. doi: 10.1371/journal.pone.0062295

[47] Cosman F, Crittenden DB, Achadi JD et al. Romosozumab treatment in postmenopausal women with osteoporosis. N Engl J Med 2016; 375 (16): 1532-1543. doi: 10.1056/NEJMoa1607948 